\title{
GRANULAR FERTILIZER MASS PREDICTION FOR ELECTRIC FERTILIZER DISTRIBUTION DEVICE BASED ON RANSAC
}

\author{
HAN, J. ${ }^{1}-$ WANG, X. T. ${ }^{1}-$ ZHOU, Z. ${ }^{1}-$ WANG, X. ${ }^{2 *}$ \\ ${ }^{1}$ Electrical and Information College, Heilongjiang Bayi Agricultural University \\ Daqing 163319, PR China \\ ${ }^{2}$ Engineering College, Heilongjiang Bayi Agricultural University, Daqing 163319, PR China \\ ${ }^{*}$ Corresponding author \\ e-mail:byndwangx@163.com \\ (Received 23 ${ }^{\text {rd }}$ Feb 2019; accepted $3^{\text {rd }}$ May 2019)
}

\begin{abstract}
To improve the precision of granular fertilizer mass measured via microwave Doppler technology, this study proposed a method based on RANSAC to predict granular fertilizer mass for fertilizer apparatus. The maximum value, the minimum value, and a random value in the sample data were selected to obtain the best-fitting line that contained most interior points using iterative method. The fitting result of LS method was also presented to compare with the proposed scheme. Simulation results and experimental results illustrate that RANSAC method has a higher prediction precision than traditional LS method and a better ability to reduce the effect of measurement error.
\end{abstract}

Keywords: microwave Doppler, sensor, solid flow and mass, electric fertilizer distribution, opening degree

\section{Introduction}

In recent years, grain system applied in agricultural production has been modernized and normalized in China. Solid flow meter is widely used in the field of variable rate fertilization, grain storage, and flour milling measurement. Online continuous measurement and the follow-up control of solid mass plays an important role in different agricultural industries, and creates a massive demand for solid flow and mass monitoring system. The precision of solid flow and mass measurement has a significant meaning on precision fertilization and energy saving. A large variety of methods used to measure solid flow and mass have been proposed in the past twenty years.

Invasive method and non-invasive method are the two common ways to measure solid flow and mass in delivery pipe (Yan and Stewart, 2000). Invasive method using apparatus with direct connection to the measuring object. Invasive instrument is regularly utilized to measure the flow of stive and corrosion without direct contact, which has a better stability, a higher precision and a longer service life. Hence the invasive method has been studied in the previous research of solid flow and mass measurement field (Zheng, 2010). Using Doppler frequency shift in the wave propagation, Doppler measurement is an invasive method, which includes laser Doppler measurement, ultrasound Doppler measurement, and photon Doppler measurement (Dimaczek et al., 1994).

Crame indicated the advantages of Doppler method applying ultrasound Doppler to different kinds of liquid metal flow measurement (Cramer et al., 2013). Shames et al. (2013) studied target location and signal of Doppler frequency shift (Shames et al., 2013; Karaboga and Latifoglu, 2013). Isa and Wu (2004) designed a solid flow 
monitoring system based on microwave Doppler radar sensor, and pointed out that the echo energy, which was transformed from solid flow signal using FFT (Fast Fourier Transform), and solid mass had a linear relation (Isa and Wu, 2004, 2006). Pang et al. (2018) investigated the relation between grain size and dielectric constant and proposed a real time invasive measurement for solid granule.

Random Sample Consensus (RANSAC) method builds a mathematics model to estimate parameters even if there is abnormal data in the data set (Hossein-Nejad and Nasri, 2016; Niedfeldt et al., 2017). Wang et al. (2016) presented a RANSAC method based on screening and matching, which has a high robustness in the environment of contamination and low computation complexity. Based on RLS model, granular mass was obtained via power spectral density in the previous studies, resulting in low sampling precision. As an iterative algorithm, RANSAC method is used to estimate the parameter of mathematics model in an outlier observation data. RANSAC method is usually applied in the field of spectral analysis and image processing. To the authors' best knowledge, RANSAC has been rarely used in the granule fertilizer mass measurement. To improve the accuracy of the granule fertilizer mass, RANSAC method is used to obtain the optimal linear function of the sample in this study.

The study focused on fertilizing mass measurement of granular fertilizer. Data set collected by Doppler sensor is the voltage of echo signal, and output of the mathematics model is the mass of granule fertilizer. A granular fertilizer applicator was developed in Heilongjiang Bayi Agricultural University to collect echo signal voltage by microwave Doppler radar sensor. The relationship of fertilizer apparatus open degree and granular fertilizer mass was investigated using Random Sample Consensus (RANSAC) algorithm. The fertilizer mass prediction model provides reference for the optimization of solid flow and mass measurement. Simulation and experimental results show that the RANSAC method used to predict the mass of granular fertilizer online has a high precision and stability. The presented method provides a scientific fundament for mass measurement of granular material, which has a great meaning of improving the grain quality and yield.

\section{Materials and methods}

\section{Materials}

In this research, this fertilizer is granular compound fertilizer diammonium phosphate, and the total nutrient of granule fertilizer: $(\mathrm{N}+\mathrm{P} 2 \mathrm{O} 5) \geq 64.0 \%$. This granular fertilizer is irregular granular and its surface is smooth, which is suitable for signal acquisition by microwave Doppler sensor.

\section{Methods of the RANSAC Algorithm for granular fertilizer mass prediction}

\section{Structure of granular fertilizer applicator}

Figure 1 shows the structure of the granular fertilizer applicator. The appliance is consisted of fertilizer box, electric fertilizer apparatus, microwave Doppler radar sensor, data collector, and a computer. Capacity of the fertilizer box is $20 \mathrm{~L}$. Power of the fertilizer apparatus is $12 \mathrm{~V} / 50 \mathrm{~W}$. Diameter of the fertilizer pipeline is $4 \mathrm{~cm}$. Microwave Doppler radar sensor is installed outside of the pipeline $30 \mathrm{~cm}$ away from the release location of the granular fertilizer. 


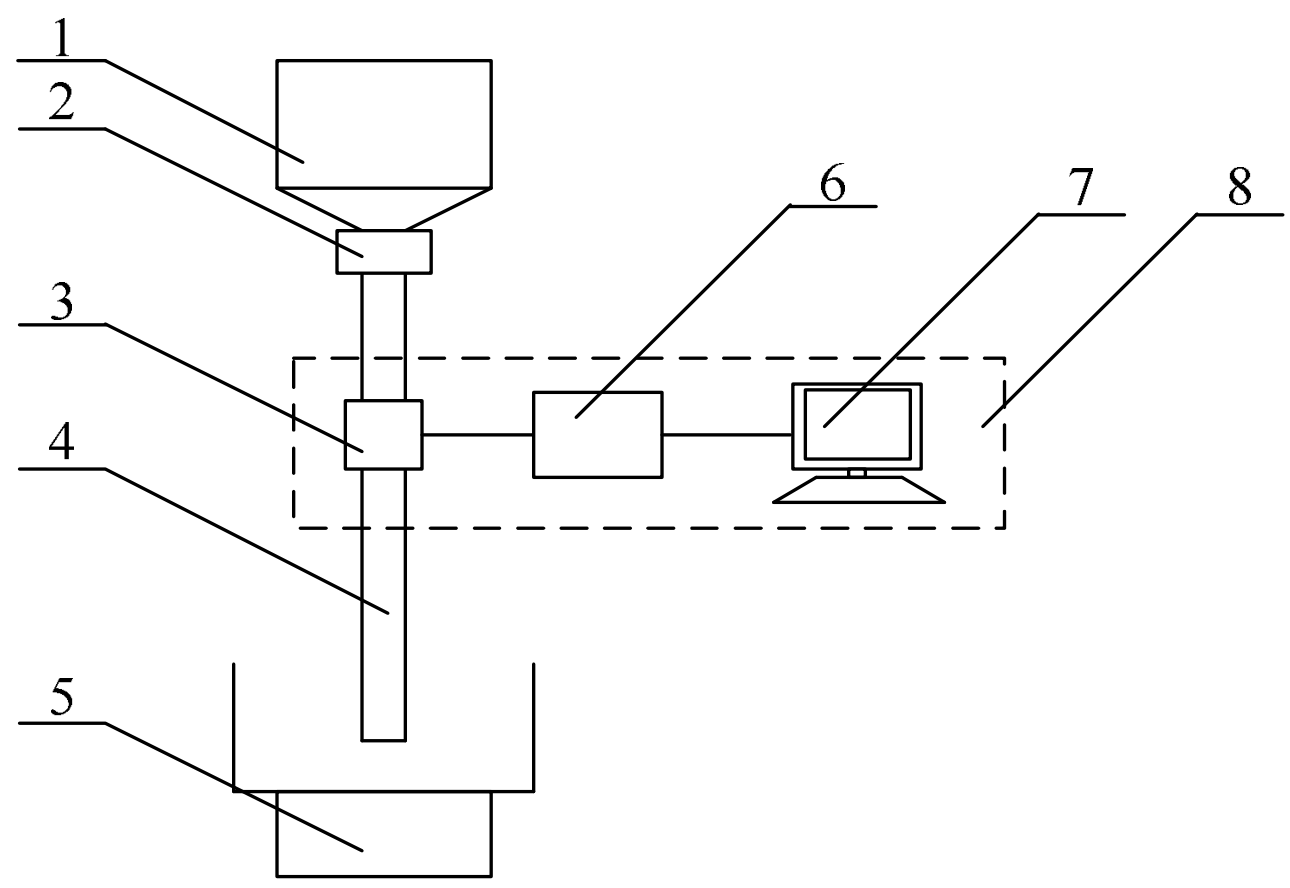

Figure 1. Structure of granular fertilizer flow measurement appliance. 1-Storage Silo, 2Electric Fertilizer Distribution Device, 3-Microwave Doppler Sensor, 4-Distributing Pipe, 5Weighing Machine, 6-Data Acquisition Unit, 7-Computer, 8-Micorwave Doppler Solid Mass Flow Measurement System

\section{Algorithm description}

For the granular fertilizer mass prediction model, voltage value of echo signal is taken as data set, unknown parameter of the sample model is granular fertilizer mass. The Voltage measured by Doppler sensor is linearly related to the opening degree of fertilizer apparatus. To improve the accuracy of the fitting line obtained by RANSAC method, the minimum value (X1, Y1), the maximum value (X2, $\mathrm{Y} 2)$, and a random value $(\mathrm{X} 3, \mathrm{Y} 3)$ are chosen in the establishment process of the initialized model. The three values are reserved in subset $M_{j}$. The optimal linear model determined by the three feature points is given as Eq.l.

$$
\mathrm{Ax}+\mathrm{By}+\mathrm{C}=0
$$

The distance from other point (Xi, Yi) to the optimal line is given as Eq.2.

$$
d_{i}=\frac{\left|A x_{i}+B y_{i}+C\right|}{\sqrt{A^{2}+B^{2}}}
$$

If $\mathrm{di}<\mathrm{dh},(\mathrm{Xi}, \mathrm{Yi})$ is inside the sample set $\mathrm{M}_{\mathrm{i}}$, otherwise is outside the sample set. The inside point set is used to estimate a new line via least square (LS) method.

$t$ is the probability of the inside point appearing in the sample set, which is expressed as Eq.3.

$$
\mathrm{t}=\frac{n_{\text {inliers }}}{n_{\text {inliers }}+n_{\text {outliners }}}
$$


The probability of at least one of the three points exiting outside the sample set is defined as Eq.4.

$$
1-t^{3}
$$

If none of the three points is inside point in $\mathrm{k}$ times random sampling, there is $\left(1-\mathrm{t}^{3}\right)^{\mathrm{k}}$. The wrong solution is obtained as Eq.5.

$$
\mathrm{P}=1-\left(1-t^{3}\right)^{k}
$$

where, $k$ is iteration step, which is the minimum number of random sampling. $P$ is the probability of obtaining the right solution.

The probability of inside point $t$ is a priori value, which has a crucial influence on the judgement of inside point and outside point. To ensure the probability $P$ of the right solution, iteration step $k$ must increase even if $t$ is large. From $E q .6, k$ can be calculated if $P$ is known.

$$
\mathrm{k}=\frac{\lg (1-p)}{\lg \left(1-t^{3}\right)}
$$

\section{Simulation results}

\section{Data acquisition}

Data acquisition instrument developed at Heilongjiang Bayi Agriculture University was employed to measure the flow of large granular urea in the field experiment. Diameter of the urea granular fertilizer used in experiment was $2.00 \mathrm{~mm}-4.75 \mathrm{~mm}$. The acquisition frequency was $500 \mathrm{~Hz}$. Echo signal voltage of the granular fertilizer flow waveform was obtained. The waveform of $10 \%, 50 \%$ and $100 \%$ opening degree is shown in Figure 2.

The voltage of each 10 percent of the fertilizer apparatus opening degree was measured for six times. Mass of each 10 percent opening degree was also measured using electronic scale for six times. The acquisition time was 10 seconds, which was counted by a time relay to improve the timing precision. Table 1 is the average mass. To indicate accuracy of the measurement, maximum error between acquisition value and average value is also presented in Table 1.

\section{Granular fertilizer mass prediction based on $L S$}

Table 2 is granular fertilizer mass in one-unit interval and quadratic sum of voltage amplitude at each $10 \%$ opening degree. $\frac{1}{n} \sum_{i=1}^{n} V^{2}$ is the average value of the echo signal voltage amplitude. Figure 3 is the fitting line of voltage amplitude average value and mass of different opening degree in one-unit interval based on LS.

The predicting equation based on LS method shown in Figure 3 is expressed as Eq.7.

$$
y=0.0885 \mathrm{x}-0.0992
$$


where, $\mathrm{y}$ is average value of the voltage amplitude, $\mathrm{x}$ is granular fertilizer mass in one unit interval. Table 3 is the absolute error between actual value and LS prediction value.

The nonlinear error is given by Eq.8.

$$
\epsilon_{\mathrm{IS}}=\frac{|(\Delta \mathrm{YL}) \mathrm{mgx}|}{\mathrm{yFs}} \times 100 \%
$$

where, $y_{F S}$ is full range output, $\Delta\left(\mathrm{y}_{\mathrm{L}}\right)_{\max }$ is the maximum deviation in the measurement points.

The maximum deviation occurred when the fertilizer apparatus opening degree was $60 \%$. According to Eq.8, the linearity degree $\epsilon_{L S}$ is $11.7 \%$.

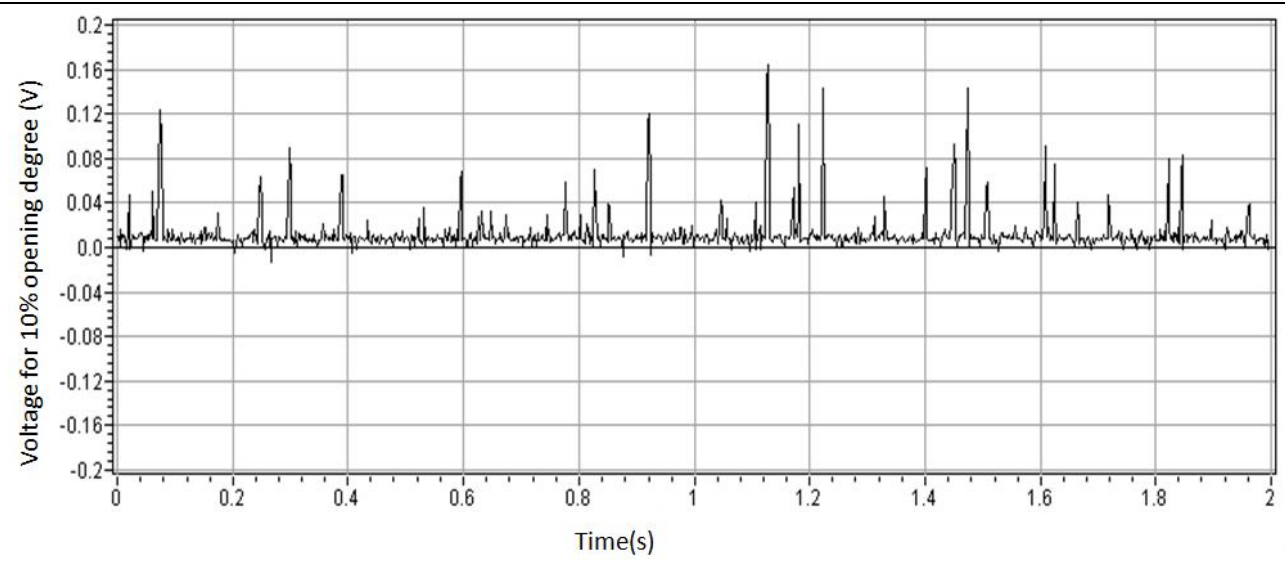

(a) $10 \%$ opening degree

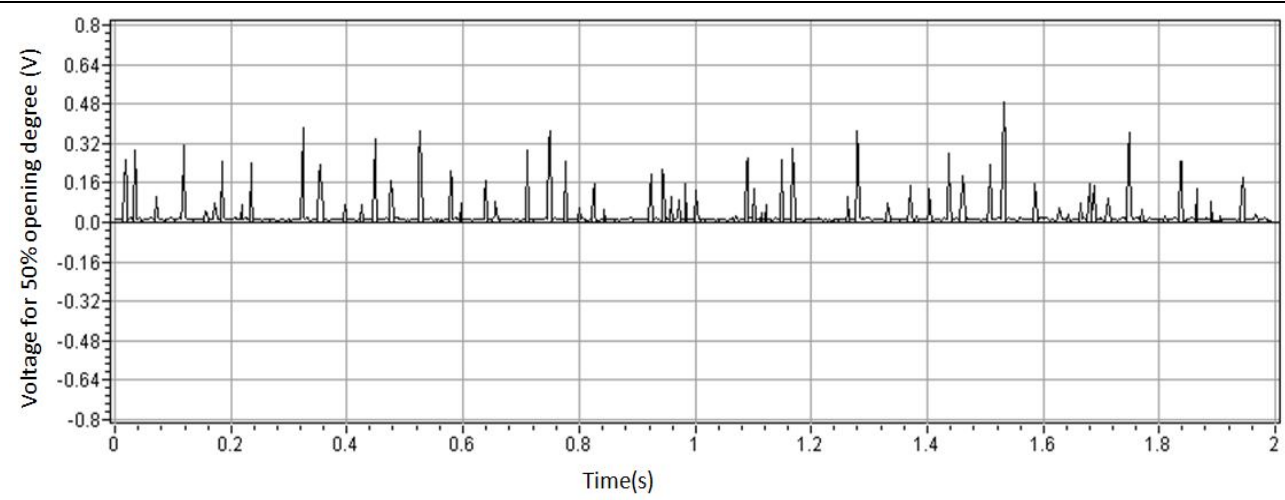

(b) $50 \%$ opening degree

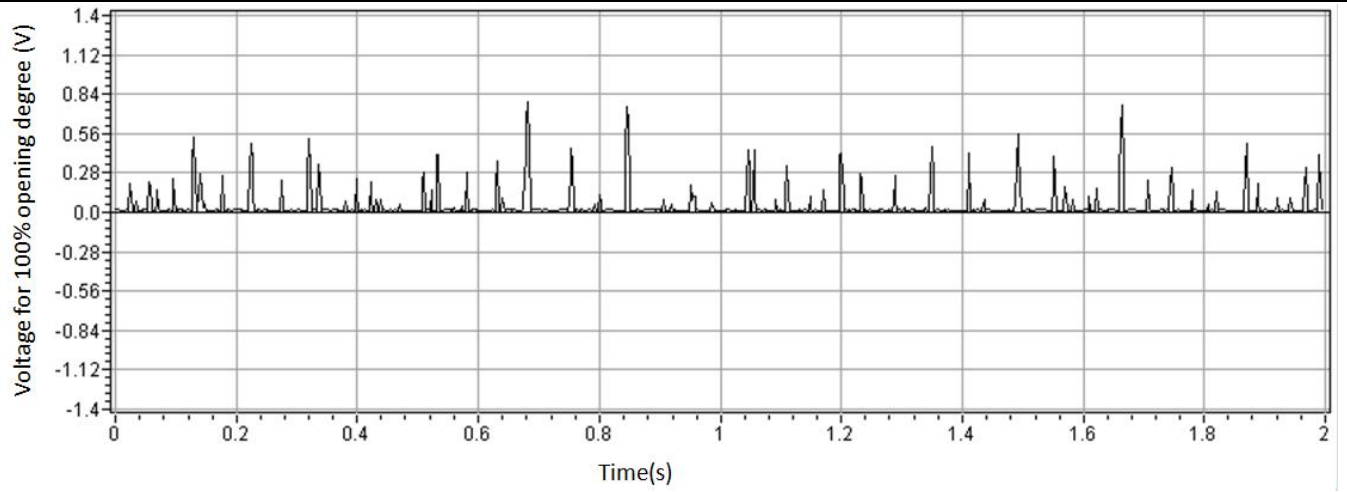

(c) $100 \%$ opening degree

Figure 2. Waveform of granular fertilizer flow voltage 
Table 1. Manual measurement statistics of granular fertilizer

\begin{tabular}{c|c|c|c|c|c|c|c|c|c|c}
\hline Opening degree & $\mathbf{1 0 \%}$ & $\mathbf{2 0 \%}$ & $\mathbf{3 0 \%}$ & $\mathbf{4 0 \%}$ & $\mathbf{5 0 \%}$ & $\mathbf{6 0 \%}$ & $\mathbf{7 0 \%}$ & $\mathbf{8 0 \%}$ & $\mathbf{9 0} \%$ & $\mathbf{1 0 0} \%$ \\
\hline Average Mass (g) & 28.55 & 135 & 215.35 & 262.37 & 327.64 & 400.8 & 467.17 & 550.83 & 601.6 & 675.32 \\
Maximum error (\%) & $7.88 \%$ & $2.96 \%$ & $2.40 \%$ & $1.46 \%$ & $1.17 \%$ & $0.85 \%$ & $1.42 \%$ & $0.48 \%$ & $0.37 \%$ & $0.91 \%$ \\
\hline
\end{tabular}

Table 2. Granular fertilizer mass and quadratic sum of the voltage amplitude

\begin{tabular}{c|c|c|c|c|c}
\hline $\begin{array}{c}\text { Opening degree } \\
(\%)\end{array}$ & $\begin{array}{c}\text { Unit time } \\
\text { mass } \mathbf{( g / s )}\end{array}$ & $\frac{1}{n} \sum_{i=1}^{n} V^{2}$ & $\begin{array}{c}\text { Opening degree } \\
\mathbf{( \% )}\end{array}$ & $\begin{array}{c}\text { Mass } \\
(\mathbf{g})\end{array}$ & $\frac{1}{n} \sum_{i=1}^{n} V^{2}$ \\
\hline $10 \%$ & 2.855 & 0.226014 & $60 \%$ & 40.08 & 3.85177 \\
$20 \%$ & 13.5 & 0.981529 & $70 \%$ & 46.71667 & 3.944421 \\
$30 \%$ & 21.535 & 1.678178 & $80 \%$ & 55.08333 & 5.102993 \\
$40 \%$ & 26.21667 & 2.191701 & $90 \%$ & 60.16 & 5.090327 \\
$50 \%$ & 32.764 & 2.703428 & $100 \%$ & 67.532 & 5.679472 \\
\hline
\end{tabular}

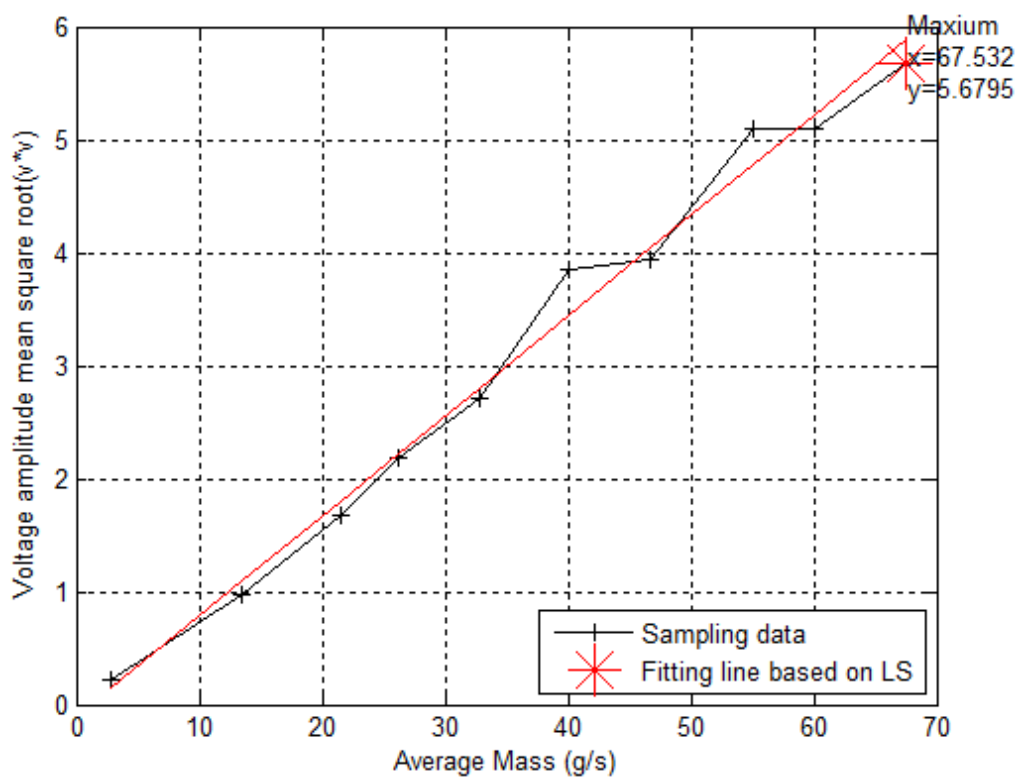

Figure 3. Fitting line based on LS

Table 3. Absolute error between actual value and LS prediction value

\begin{tabular}{c|c|c|c|c}
\hline $\begin{array}{c}\text { Fertilizer apparatus } \\
\text { opening degree }\end{array}$ & $\begin{array}{c}\text { Granular fertilizer } \\
\text { mass (g) }\end{array}$ & $\frac{1}{n} \sum_{i=1}^{n} V^{2}$ & $\begin{array}{c}\text { Sampling point of LS } \\
\text { linear equation }\end{array}$ & Error \\
\hline $10 \%$ & 2.855 & 0.2260138 & 0.1535 & 0.072514 \\
$20 \%$ & 13.5 & 0.978848294 & 1.0959 & -0.11705 \\
$30 \%$ & 21.535 & 1.678178 & 1.8072 & -0.12902 \\
$40 \%$ & 26.21667 & 2.191701 & 2.2216 & -0.0299 \\
$50 \%$ & 32.764 & 2.703428 & 2.8012 & -0.09777 \\
$60 \%$ & 40.08 & 3.85177 & 3.4489 & 0.40287 \\
$70 \%$ & 46.7166 & 3.944421 & 4.0364 & -0.09198 \\
$80 \%$ & 55.08333 & 5.102993 & 4.7770 & 0.325993 \\
$90 \%$ & 60.16 & 5.090327 & 5.2265 & -0.13617 \\
$100 \%$ & 67.532 & 5.679472 & 5.8791 & -0.19963 \\
\hline
\end{tabular}




\section{Granular fertilizer mass prediction based on RANSAC}

Figure 4 is the fitting line of voltage amplitude average value and mass of different opening degree in one-unit interval based on RANSAC. Table 4 is the absolute error between actual value and RANSAC prediction value.

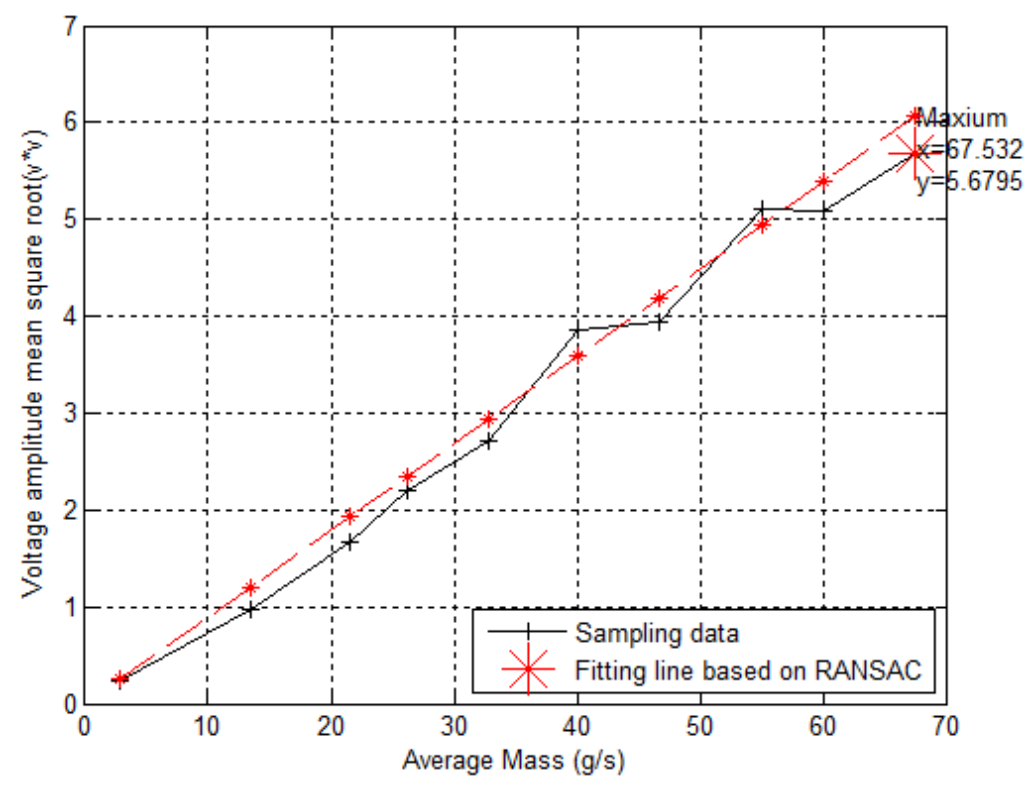

Figure 4. Fitting line based on RANSAC

Table 4. Absolute error between actual value and RANSAC prediction value

\begin{tabular}{c|c|c|c|c}
\hline $\begin{array}{c}\text { Fertilizer apparatus } \\
\text { opening degree }\end{array}$ & $\begin{array}{c}\text { Granular fertilizer } \\
\text { mass (g) }\end{array}$ & $\frac{1}{n} \sum_{i=1}^{n} V^{2}$ & $\begin{array}{c}\text { Sampling point of } \\
\text { RANSAC linear } \\
\text { equation }\end{array}$ & Error \\
\hline $10 \%$ & 2.855 & 0.2260138 & 0.2464 & -0.02039 \\
$20 \%$ & 13.5 & 0.978848294 & 1.2038 & -0.22495 \\
$30 \%$ & 21.535 & 1.678178 & 1.9265 & -0.24832 \\
$40 \%$ & 26.21667 & 2.191701 & 2.3476 & -0.1559 \\
$50 \%$ & 32.764 & 2.703428 & 2.9365 & -0.23307 \\
$60 \%$ & 40.08 & 3.85177 & 3.5946 & 0.25717 \\
$70 \%$ & 46.7166 & 3.944421 & 4.1915 & -0.24708 \\
$80 \%$ & 55.08333 & 5.102993 & 4.9441 & 0.158893 \\
$90 \%$ & 60.16 & 5.090327 & 5.4007 & -0.31037 \\
$100 \%$ & 67.532 & 5.679472 & 6.0637 & -0.38423 \\
\hline
\end{tabular}

The predicting equation based on RANSAC method shown in Figure 5 is defined as Eq.9.

$$
y=0.089945 \mathrm{x}-0.010418
$$

The maximum deviation occurred when the fertilizer apparatus opening degree was $100 \%$. According to Eq.9, the nonlinear error $\epsilon_{L S}$ is $6.34 \%$.

The nonlinear error of the RANSAC line, which is less than the nonlinear error of LS. To obtained a minimum sum of squared error, if there is large deviation point, the error 
of fitting method based on LS is large. As regards to RANSAC method, the points near the fitting line are selected by eliminating the large deviation point. Hence, the prediction accuracy with RANSAC is higher than LS.

\section{Result}

Figure 5 is the comparison of the actual value and prediction value. The well-fitted line demonstrates the effectiveness of the proposed RANSAC method. which is better fitting than the LS line. Table 5 is the result of MSE and nonlinear error with RANSAC and LS method.

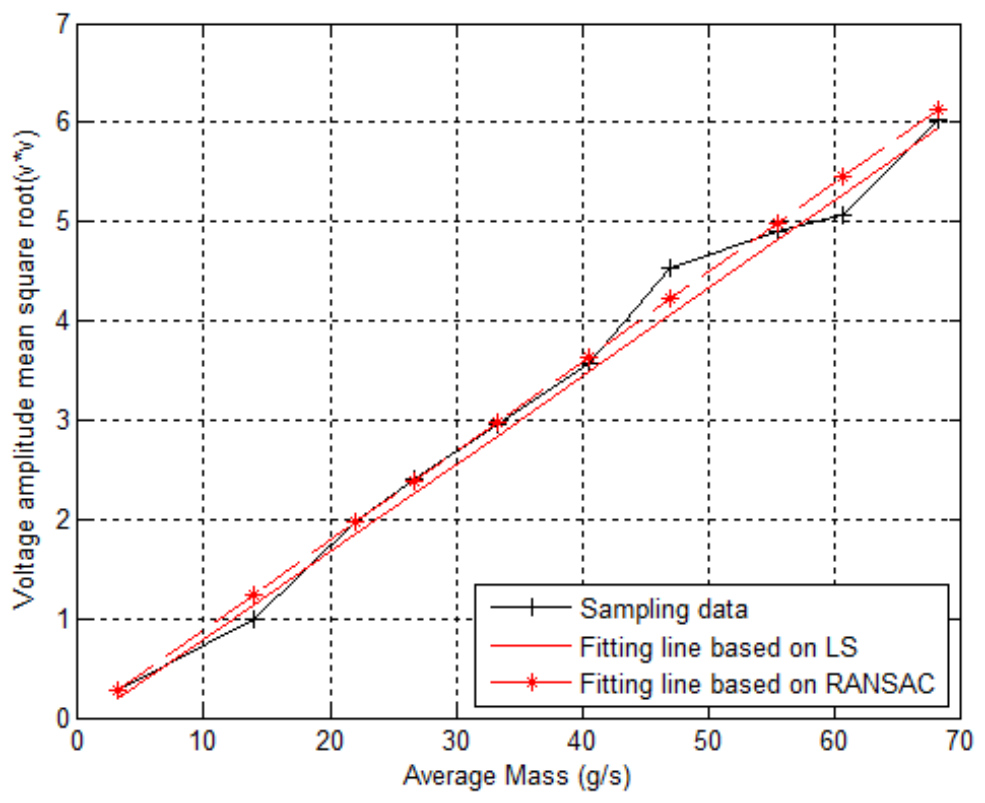

Figure 5. Fitting lines based on RANSAC and LS

Table 5. Results of MSE and nonlinear error

\begin{tabular}{c|c|c}
\hline Fitting method & MSE & Nonlinear error \\
\hline LS & 0.45702 & $11.2 \%$ \\
RANSAC & 0.37787 & $5.43 \%$ \\
\hline
\end{tabular}

\section{Conclusion and discussion}

The echo signal voltage of urea granular fertilizer was measured by Doppler sensor. The prediction model of granular fertilizer mass based on RANSAC was built. The proposed method was compared with LS algorithm. Simulation results demonstrate that the prediction accuracy with RANSAC is higher than LS.

The LS method considers the minimum square error of all location points in linear fitting. When there are several points with larger deviation, the fitting error is larger. Using RANSAC to estimate the Doppler signal, we can select consistent sets that conform to the straight line when fitting straight lines, automatically eliminate the larger error points, and improve the fitting accuracy. Subsequent research will further improve signal sampling accuracy, reduce computation time and improve operational efficiency. 
Acknowledgements. This work was supported by 13th Five-year Plan of National Key Research and Development Project (NO. 2016YFD020060802).

\section{REFERENCES}

[1] Cramer, A., Eckert, S., Gerbeth, G. (2013): Flow measurements in liquid metals by means of ultrasonic Doppler method and local potential probes. - European Physical Journal Special Topics 220: 25-41.

[2] Dimaczek, G., Fassbinder, H.-G., Emmel, A., Kupfer, R. (1994): High-precision Coriolis mass flowmeter for bulk material two-phase flows. - Flow Measurement \& Instrumentation 5: 295-302.

[3] Hossein-Nejad, Z., Nasri, M. (2016): Image registration based on SIFT features and adaptive RANSAC transform. - International Conference on Communication and Signal Processing. IEEE.

[4] Hossein-Nejad, Z., Nasri, M. (2018): A-RANSAC: Adaptive random sample consensus method in multimodal retinal image registration. - Biomedical Signal Processing and Control 45: 325-338.

[5] Isa, M. M., Wu, Z. Q. (2004): Microwave radar sensor as solid flow counter. - High Frequency Postgraduate Student Colloquium. IEEE: 37-40.

[6] Isa, M. M., Wu, Z. Q. (2006): Microwave Doppler Radar Sensor for Solid Flow Measurements. - Eruopean microwave Conference. IEEE: 1508-1510.

[7] Karaboga, N., Latifoglu, F. (2013): Elimination of noise on transcranial Doppler signal using IIR filters designed with bee colony-ABC -algorithm. - Digital Signal Processing: 1051-1058.

[8] Le, V.-H., Vu, H., Nguyen, T. T., Le, T.-L., Tran, T.-H. (2018): Acquiring qualified samples for RNSAC using geometrical constraints. - Pattern Recognition Letters 102: 5866.

[9] Niedfeldt, P. C., Ingersoll, K., Beard, R. W. (2017): Comparison and analysis of recursive-RANSAC for multiple target tracking. - IEEE Transactions on Aerospace \& Electronic Systems PP (99): 1-1.

[10] Pang, L., Shao, Y., Geng, C., Zhong, W., Liu, G., Liu, L., Tian, W. (2018): Measurement of solid mass flow rate by a non-intrusive microwave method. - Powder Technology 323: 525-532.

[11] Shames, I., Bishop, A. N., Smith, M., Anderson, B. D. O. (2013): Doppler shift target localization. - IEEE Transactions on Aerospace and Electronic Systems 49: 266-276.

[12] Wu, Z., Wang, H. G., Isa, M., Liu, C. G. (2014): Laboratory evaluation of microwave Doppler velocimeter for solid flow measurements. - American Institute of Physics Conference Series. American Institute of Physics: 57-62.

[13] Xu, J. J., Huang, L., Yin, S., Gao, B., Chen, P. (2018): All-fiber self-mixing interferometer for displacement measurement based on the quadrature demodulation technique. - Opt Rev. 25(1): 40-45.

[14] Yan, Y., Stewart, D. (2000): Flow measurement of particulate solids in pipelines. - Flow Measurement \& Instrumentation 11: 151-151.

[15] Zhang, N.-B., Xu, J.-J., Xue, C.-G. (2011): Core-shell structured mesoporous silica nanoparticles equipped with pyrene-based chemosensor: Synthesis, characterization, and sensing activity towards Hg(II). - Journal of Luminescence 131: 2021-2025.

[16] Zhu, L., Xu, J., Yan, L. (2017): Research on congestion elimination method of circuit overload and transmission congestion in the internet of things. - Multimedia Tools and Applications 76: 18047-18066. 